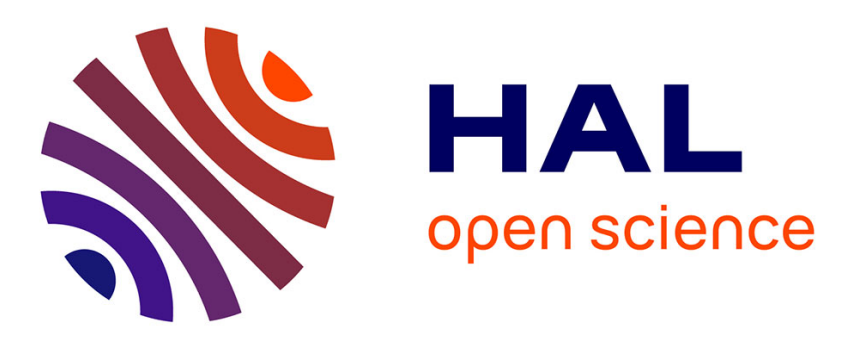

\title{
Beyond the linear and Duffing regimes in nanomechanics: Circularly polarized mechanical resonances of nanocantilevers
}

S. Perisanu, Thomas Barois, A. Ayari, P. Poncharal, M. Choueib, S. T. Purcell, P. Vincent

\section{To cite this version:}

S. Perisanu, Thomas Barois, A. Ayari, P. Poncharal, M. Choueib, et al.. Beyond the linear and Duffing regimes in nanomechanics: Circularly polarized mechanical resonances of nanocantilevers. Physical Review B: Condensed Matter and Materials Physics (1998-2015), 2010, 81 (16), pp.165440. 10.1103/PhysRevB.81.165440 . hal-01565122

\section{HAL Id: hal-01565122 \\ https://hal.science/hal-01565122}

Submitted on 5 Oct 2017

HAL is a multi-disciplinary open access archive for the deposit and dissemination of scientific research documents, whether they are published or not. The documents may come from teaching and research institutions in France or abroad, or from public or private research centers.
L'archive ouverte pluridisciplinaire HAL, est destinée au dépôt et à la diffusion de documents scientifiques de niveau recherche, publiés ou non, émanant des établissements d'enseignement et de recherche français ou étrangers, des laboratoires publics ou privés. 


\title{
Beyond the linear and Duffing regimes in nanomechanics: Circularly polarized mechanical resonances of nanocantilevers
}

\author{
S. Perisanu, ${ }^{*}$ T. Barois, A. Ayari, P. Poncharal, M. Choueib, S. T. Purcell, and P. Vincent ${ }^{\dagger}$ \\ LPMCN, Université Claude Bernard Lyon 1 et CNRS, UMR 5586, F-69622 Villeurbanne, France
}

(Received 6 April 2010; published 28 April 2010)

\begin{abstract}
We present here an experimental and theoretical study of the nonlinear coupling between the two polarizations of the mechanical resonances of singly clamped nanowires and nanotubes, leading to circularly polarized oscillations. This regime exists for high amplitudes and beyond the frequency range of the "classical" Duffing regime. Good quantitative agreement is found with a simple theoretical model based on the first nonlinear terms of the coupling. The assumptions used in the model are quite general and thus the circular movement is a universal response for nanocantilevers.
\end{abstract}

DOI: $10.1103 /$ PhysRevB.81.165440

PACS number(s): 85.85.+j, 05.45.-a, 62.25.Jk, 63.22.Gh

\section{INTRODUCTION}

Nanotubes and nanowires (NNs) are presently attracting considerable attention as resonant rods in nanoelectromechanical systems (NEMS), mainly because their extremely small physical dimensions imply high sensitivity to external perturbations and open perspectives for measuring extremely weak signals. This is in addition to the general advantage of NEMS having much higher quality factors ${ }^{1,2}$ than purely electronic devices (see Fig. 1D in Ref. 3). An example of the potential of NNs in sensing is atomic mass resolution measurements. ${ }^{4-6}$ However, the NNs high sensitivity implies that nonlinear response with its complicated signature of jumps and hysteresis occurs for relatively low external perturbations, thus limiting straightforward use of measured signals. This is a real bottleneck to the integration of NEMS devices.

Another approach is to try to exploit the rich nonlinearresponse phenomena. Examples are using the jumps for measuring fine frequency shifts, ${ }^{7}$ generating self-oscillations ${ }^{8}$ or single electron shuttle transport. ${ }^{9}$ A deep understanding of both experimental and theoretical aspects of the NEMS nonlinear behavior is critical for device integration and fundamental studies, such as the effects of the charge quantization. ${ }^{10,11}$ The main origin of nonlinear effects for the doubly clamped configuration is the supplementary stress due to global strain during oscillation. ${ }^{12}$ However the origin of the nonlinear effects observed in the singly clamped configuration $^{2}$ is still unclear. Moreover, there appears to be no experimental studies in the literature of nanocantilevers exhibiting two orthogonal polarizations with coupling that can lead to nonplanar motion.

In this paper we report experimental characterization of a circular polarization (CP) for the mechanical eigenmodes in the singly clamped configuration. This was observed by field emission microscopy (FEM) which gives directly a magnified image of the apex trajectory. CP takes over at higher amplitude or frequency from the "classical" Duffing regime with linear polarization (LP). A semianalytical model, found to be in excellent agreement with experiment, describes both the LP and CP using basic elements of cylindrical NNs, which means that this behavior is a universal third regime beyond the linear and Duffing regimes. This nonplanar mo- tion is a manifestation of a general phenomenon in physics where an oscillator driven at high amplitude pumps energy into unexcited degrees of freedom through nonlinear coupling.

Such nonlinear behavior was theoretically investigated for both singly and doubly clamped configurations ${ }^{13-15}$ and was previously only reported, to our knowledge, on macroscopic systems. ${ }^{16}$ There are two main reasons for this. First, this CP is not easy to observe experimentally since the commonly used motion detection techniques for NNs (capacitive and magnetomotive) do not distinguish polarizations. Second, as we will see later in this paper, in order to have CP one needs excitation amplitudes so high that they can destroy the NN.

\section{EXPERIMENTAL SETUP AND RESULTS}

Our samples were multiwall carbon nanotubes (MWNTs) and silicon carbide nanowires (SiC-NWs) individually mounted on the apexes of etched tungsten tips by nanomanipulation, ${ }^{17}$ inserted either into a FEM or a transmission electron microscope (TEM) and excited capacitively by ac signals $V_{\mathrm{ac}} \cos \left(2 \pi \nu_{\mathrm{ac}} t\right)$ on quadrupole anodes placed several millimeters away from the sample (Fig. 1). In the TEM configuration one visualizes resonating NNs nearly side on [Fig. 2(a)]. In the FEM configuration the tip with the $\mathrm{NN}$ is placed in an ultrahigh vacuum chamber and brought to a negative voltage of a few hundreds of volts with respect to a phosphor screen. Electrons tunnel from the NN apex and strike the phosphor screen to form a FEM pattern [Fig. 2(b)]. When a resonance of an $\mathrm{NN}$ is excited the patterns enlarge parallel to the motion ${ }^{18,19}$ and we observe an averaged image because of our standard $25 \mathrm{~Hz}$ video camera. The FEM pattern increase is detected with high sensitivity through an image analysis method. ${ }^{17}$ In both experiments all potentials were applied on macroscopical elements placed at least a few millimeter away from the NN, which means we can neglect the electric field variation with the NN displacement. The main strength of FEM for this experiment is that it allows us to visualize accurately the NN nonplanar motion since the $X-Y$ FEM image plane is perpendicular to the NN axis, compared to the TEM configuration where the NN lies almost in the image plane, thus obscuring one direction of motion. 


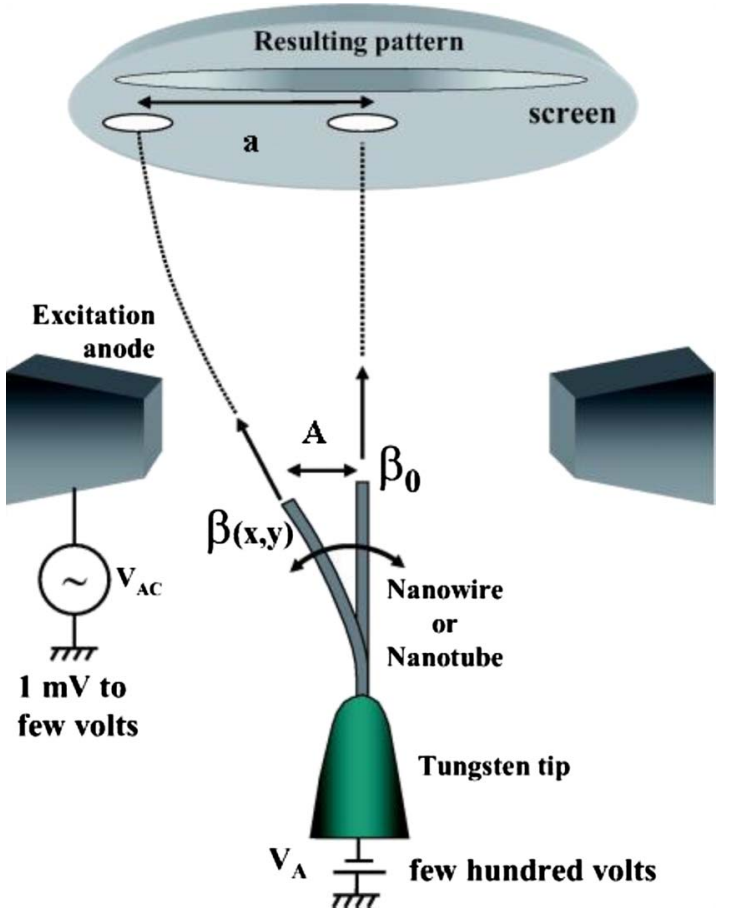

FIG. 1. (Color online) Schematic view of our field emission setup. The electric field generated by the $V_{A}$ is amplified at the $\mathrm{NN}$ apex by tip effect and extracts electrons from the sample. In this configuration the FE pattern on the screen is a magnified image of the nanowire apex trajectory since the $25 \mathrm{~Hz}$ standard video camera only gives the averaged motion.

Lorentzian response with $Q$ factors in the $10^{3}-10^{4}$ range was observed when scanning at low $V_{c}[Q \simeq 2000$ for the SiC-NW in Figs. 2(b)-2(i)]. An essential point is that we always observed a splitting in resonance frequencies of a few percent between the two LPs which greatly exceeds their natural Lorentzian widths, due to symmetry-breaking imperfections. We adopt the nomenclature $\nu_{n x}$ and $\nu_{n y}$ for the higher and lower $n$th mode polarizations with amplitudes $A_{n x}$ and $A_{n y}$, respectively.

Duffing type, linearly polarized, nonlinear response was observed for all the modes upon increasing $V_{c}$, as can be seen for the first mode in Fig. 2 and for the second mode in Fig. 3. The first (second) mode has a hard (soft) spring behavior, which means increasing (decreasing) of the eigenfrequency with the amplitude of oscillation and jumps at frequencies higher (lower) than the mode's Lorentzian eigenfrequency, as shown in Figs. 2 and 3.

The key moment in our experiments occurred at even higher $V_{c}$. We clearly observed nonplanar motion on the first mode, as it can be seen in the FEM and TEM images in Fig. 2. This new regime takes over from the Duffing regime at the high end of increasing frequency scans at $\nu_{1 x}$. The rest of this paper is dedicated to the understanding and characterization of this phenomenon.

Let us examine the data in more detail for later comparisons with the simulations. First, the nonplanar motion was always excited close to $\nu_{n x}$ and not $\nu_{n y}$. Second, when a NN in the Duffing regime enters the nonplanar motion regime, $A_{y}$ increases more rapidly with $\nu_{c}$ than $A_{x}$. Third, the relative
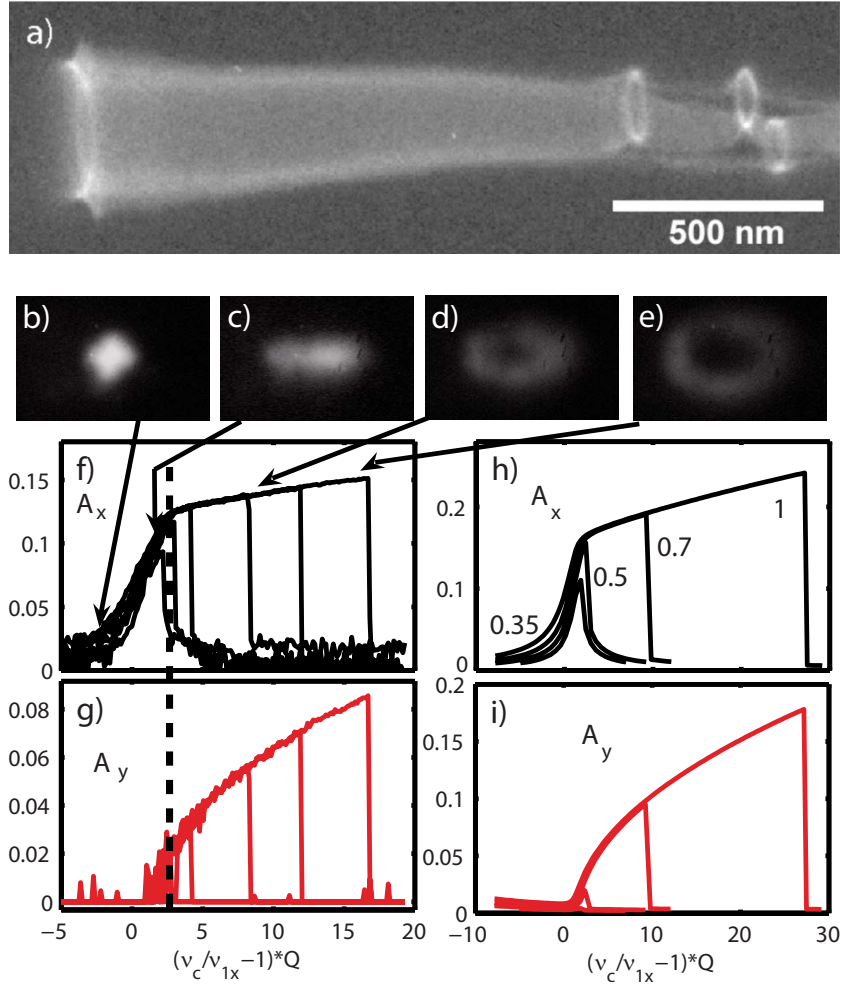

FIG. 2. (Color online) Elliptical response of the first eigenmode: (a) TEM image showing time averaged response of a MWNT with the NN end tracing out an elliptical movement. (Several defects along the MWNT also trace out ellipses.) [(b)-(e)] FEM images showing response of an SiC-NW (7 $\mu \mathrm{m}$ length and $50 \mathrm{~nm}$ diameter) to increasing excitation. First we observe the linear polarization in the horizontal $x$ direction, then the system transits to an elliptical polarization which becomes more circular with increasing excitation. [(f) and (g)] Experimental and [(h) and (i)] simulated frequency responses for $A_{x}$ and $A_{y}$, for increasing excitation amplitude near the $x$ polarization eigenfrequency $\left(\nu_{1 y}=0.989 \nu_{1 x}\right.$ $=1.527 \mathrm{MHz}$ ). The excitation forces normalized to $E I / L^{2}$ were $0.35,0.5,0.7$, and 1 for the simulated data. $A_{x}$ is Lorentzian for low excitation and hard spring nonlinear for intermediate excitation. At high $V_{\mathrm{ac}}$ and $\nu_{\mathrm{ac}}$ one observes hard spring elliptically polarized response with the eccentricity decreasing with $\nu_{\text {ac }}$ until circular polarization is almost reached, delimited in (f) and ( $\mathrm{g}$ ) by the vertical dashed line. Excellent agreement can be seen between the measured and the simulated data.

phase between the $x$ and $y$ oscillations is always $\pi / 2$. These last two mean that the NN's apex has an elliptic trajectory whose eccentricity diminishes with the applied frequency until it becomes circular, as shown on the experimental films. ${ }^{19}$ For one film we have applied an additional ac voltage to the screen to visualize the motion by stroboscopy. Note that our excitation method imposes in-phase driving for both directions, yet we observe a constant $\pi / 2$ phase difference for their response while scanning $\nu_{c}$, which is, together with the jumps, a clear signature of nonlinear coupling between the $x$ and $y$ motion. Those features cannot be explained by two uncoupled or linearly coupled polarizations with slightly different eigenfrequencies.

The same kind of nonplanar motion was also observed on the second mode [Fig. 3(a)]. When scanning the resonance at 


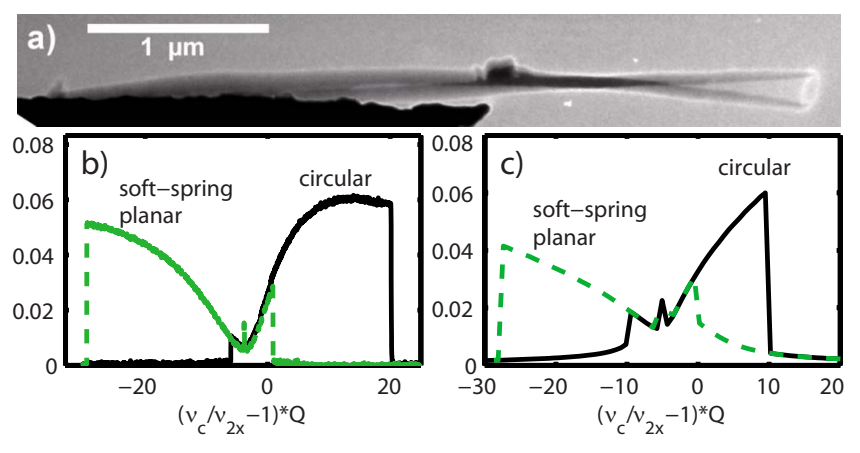

FIG. 3. (Color online) Elliptical response of the second eigenmode. (a) TEM image showing time averaged response. (b) Experimental FEM and (c) simulated response defined as $A_{r}=\sqrt{A_{x}^{2}+A_{y}^{2}}$ for increasing (-) and decreasing (- -) frequency for scans that span $\nu_{2 x}$ and $\nu_{2 y}$. One observes linearly polarized soft spring, nonlinear response for the lower frequency $y$ polarization and elliptically polarized, hard spring response for the higher frequency $x$ polarization. The shape of the experimental response is slightly altered by the size of our phosphor screen, which was not wide enough to contain the whole FEM pattern during the high amplitude oscillation, i.e., the apparent saturation is partly an artifact.

high $V_{\text {ac }}$ and increasing frequency through both its LPs, we first observed the Duffing "soft spring" behavior on $\nu_{2 y}$ and then a CP on $\nu_{2 x}$ with a "hard spring" behavior [Fig. 3(b)]. A decreasing frequency sweep showed hysteretic behavior in addition.

\section{MODELING AND DISCUSSION}

The motion of the singly clamped cylindrical rod in the linear regime is a textbook problem ${ }^{20}$ and it consists of a sequence of eigenmodes, each one having two perpendicular LPs at the same frequency. The nonlinear and nonplanar motion of the singly clamped rods were also investigated ${ }^{13}$ but we found no clear indication of the existence of CP. We will now give a simple description of the physics we observe by taking into account the influence of first nonlinear order terms. In the beginning we will not consider the frequency difference between $\nu_{n x}$ and $\nu_{n y}$, the damping and the excitation. We will include afterward all those aspects into a numerical model to describe the experimental results.

The kinetic and the potential energies of a singly clamped rod in pure flexion are given by ${ }^{20}$

$$
\begin{gathered}
K=\frac{\rho C L^{3}}{2} \int_{0}^{1}\left(\dot{x}^{2}+\dot{y}^{2}+\dot{z}^{2}\right) d s, \\
P=\frac{E I}{2 L} \int_{0}^{1}\left(x^{\prime \prime 2}+y^{\prime \prime 2}+z^{\prime \prime 2}\right) d s
\end{gathered}
$$

with $L$ the rod's length, $C$ its surface area, $I$ the area moment of inertia, $E$ the Young's modulus, and $\rho$ the density. $x(s, t)$, $y(s, t)$, and $z(s, t)$ are the coordinates at the position $s$ along the rod at time $t$ and the "dots" ("primes") represent $t(s)$ derivatives. All the lengths are normalized to $L$ and $z$ is the direction of the unbent rod.
The condition for a constant length rod is $x^{\prime 2}+y^{\prime 2}+z^{\prime 2}$ $=1$ and this generates all the nonlinear terms since Eqs. (1) and (2) have only quadratic terms and thus only generate linear response in the equations of motion.

If we put $x(s, t)=X(t) f(s)$ and $y(s, t)=Y(t) f(s)$ with $X(t)$ and $Y(t)$ generalized coordinates, $f(s)$ the linear shape of the mode $^{20}$ and we keep the first non linear terms (fourth order for the energy) we get

$$
\begin{gathered}
K=\frac{\rho C L^{3}}{2}\left[m_{1}\left(\dot{X}^{2}+\dot{Y}^{2}\right)+m_{2}(X \dot{X}+Y \dot{Y})^{2}\right], \\
P=\frac{E I}{2 L}\left[k_{1}\left(X^{2}+Y^{2}\right)+k_{2}\left(X^{2}+Y^{2}\right)^{2}\right]
\end{gathered}
$$

with constants $m_{1}, m_{2}, k_{1}$, and $k_{2}$ given in Ref. 21 .

By solving the Lagrangian equations using Eqs. (3) and (4) and normalizing the time to $\sqrt{\rho C L^{4} / E I}$ we get

$$
\ddot{W}+\frac{k_{1}+2 k_{2}\left(X^{2}+Y^{2}\right)+m_{2}\left(\dot{X}^{2}+\dot{Y}^{2}\right)}{m_{1}+m_{2}\left(X^{2}+Y^{2}\right)} W=0,
$$

where $W$ is either of $X$ or $Y$.

If we consider just the $X$ LP we get the Duffing nonlinear regime, with a dependence frequency amplitude,

$$
\omega^{2}=\frac{k_{1}}{m_{1}}\left[1+\frac{A^{2}}{2}\left(\frac{3 k_{2}}{k_{1}}-\frac{m_{2}}{m_{1}}\right)\right] .
$$

The first eigenmode has a hard spring behavior, characterized by a moderate increase in the eigenfrequency with the amplitude since $3 k_{2} / k_{1}=1.2267$ and $m_{2} / m_{1}=1.1492$. For the higher modes $3 k_{2} / k_{1}<m_{2} / m_{1}$ (10.36 compared to 36.18 for the second mode), giving an important soft spring behavior, as previously predicted in Ref. 13 and observed experimentally on a large number of samples (see Figs. 2 and 3). The relative importance of the nonlinear terms on the second mode explains why we have never observed Lorenzian response on higher order modes. The $A(\omega)$ dependence in Eq. (6) can be linearly expanded around a mode's eigenfrequency to obtain the slope of the Duffing regime [zone of Fig. 2(c) in Fig. 2(f)].

We write now Eq. (5) in polar coordinates $X=R \cos \phi$ and $Y=R \sin \phi$ for studying $\mathrm{CP}$, which gives angular momentum $R^{2} \dot{\phi}$ conservation and

$$
m_{1}\left(\ddot{R}-R \dot{\phi}^{2}\right)+m_{2} R\left(\dot{R}^{2}+R \ddot{R}\right)+k_{1} R+2 k_{2} R^{3}=0 .
$$

Those two equations admit a solution with constant $\omega=\dot{\phi}$ and constant $R$, related by

$$
\omega^{2}=\frac{k_{1}}{m_{1}}\left(1+\frac{2 k_{2}}{k_{1}} R^{2}\right) .
$$

This CP exists close to the mode's eigenfrequency and has a hard spring behavior regardless of the nonlinear behavior of the mode it comes from since $k_{2} / k_{1}$ is always positive. In fact, for this constant $R$ polarization, no soft spring nonlinear term comes from the kinetic energy since the shape of the wire is preserved during the oscillation. This is exactly the behavior we observe experimentally, as it can clearly be seen 
in Figs. 2 and 3. As for the LP nonlinear regime, we can use the first-order expansion of $R(\omega)$ in Eq. (8) to obtain the slope of the CP nonlinear regime [see zones $d$ and $e$ in Fig. 2(f)].

We can now add to Eq. (5) a $\gamma \dot{W}$ dissipation term, an $F_{W} \cos \omega t$ driving, and a $k_{1 Y}<k_{1 X} \cdot \gamma, k_{1 Y} / k_{1 X}$, and $F_{Y} / F_{X}$ were adjusted to match the experimental values for the first (second) mode of the quality factor $Q=2000(Q \simeq 3000)$, of the eigenfrequency shift $S=\left(\nu_{1 x}-\nu_{1 y}\right) / \nu_{1 x}=1.1 \%$ $(S \simeq 0.5-0.7 \%)$, and of the Lorentzian (almost Lorentzian) response amplitude ratio for the LPs $\simeq 1(\simeq 0.5)$. Results of the numerical simulation are in excellent agreement with experimental data, as shown in Figs. 2 and 3 and in the simulated film. ${ }^{19}$ For example, in the case of $\mathrm{CP}$, this numerical model clearly describes the appearance of ellipses only around $\nu_{n x}$ and never around $\nu_{n y}$ together with the decreasing of the eccentricity with $\nu_{c}$ in addition to the $\pi / 2$ constant phase between the $X$ and $Y$ oscillations and the hard spring behavior independent of the mode, which were already explained by the analytical model.

The reason why we can clearly observe this $\mathrm{CP}$ is that it survives in a large frequency range where the LP Duffing regime vanishes or becomes unstable. In both nonlinear regimes the frequency at which the jumps occur is determined by the external driving and by the dissipation while the amplitude of oscillation is only determined by the nonlinear terms in the equation of motion and is almost independent of the external driving, as we can see in Figs. 2(f)-2(i). Simulations for the first mode gave a range of frequency much larger for the CP than for the LPs for high driving, as we can see in Fig. 4(a). This behavior is consistent with experimental observations presented in Fig. 2. For the second mode, the soft spring LP Duffing regime does not overlap with the hard spring CP.

Together with the excitation frequency, two other critical parameters for the existence of nonplanar modes are the driving amplitude $F$ and the eigenfrequency shift $S$. We present in Fig. 4(b) a phase diagram showing the zone of nonplanar motion existence in the $S-F$ plane. We can see that for $S$ $\simeq 1 \%$ we need an excitation $F \simeq 0.5$ and thus, using Fig. 2, $A_{1 x} / L \simeq 0.17$ for the nonplanar motion to appear on the first mode.

This high threshold for CP excitation is one reason why this mode has never been reported for NN resonances (to our knowledge), despite the considerable work done in this subject. ${ }^{12,22-25}$ In our own experiments, even if we reproducibly saw the CP on at least ten samples, they did not represent more than $20 \%$ of the studied samples. We commonly have $S>1.5 \%$ and thus, according to Fig. 4(b), we need
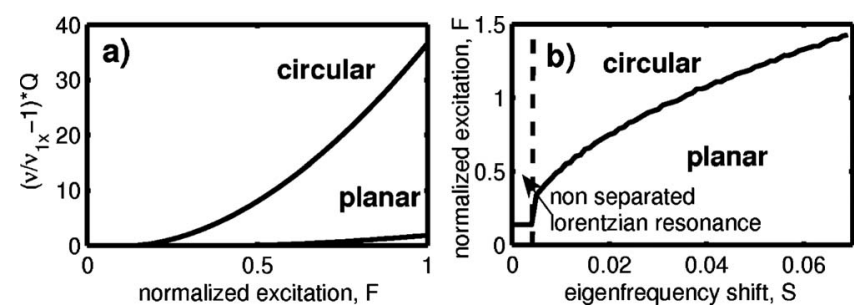

FIG. 4. For the first mode: (a) calculated normalized bistability width of the resonance as a function of the excitation amplitude. We can see the CP survives in a frequency range much larger than the LP. This width slightly exceeds the simulated one in Figs. 2(g) and 2(h), which gives us an idea of the numerical uncertainties in the simulation. (b) Simulated excitation amplitude versus eigenfrequency shift phase diagram of the $\mathrm{CP}$ existence. Comparison with Fig. 2 shows us we need amplitudes around 20\% of the wire's length to get $\mathrm{CP}$.

$A_{1 x} / L>20 \%$ to obtain CP on the first mode. Such important oscillation amplitudes can completely unglue or destroy the NN sample and thus severely limit the possibility to observe CP. We can decrease this threshold amplitude by fabricating NNs with less geometrical defects and thus smaller $S$ but this is beyond the scope of this paper.

\section{CONCLUSION}

We have presented experimental evidence for the existence of a nonlinear mechanical circular polarization for the modes of singly clamped NNs. A theoretical model including nonlinear terms up to the fourth order in the Lagrangian gives excellent agreement with experimental observations. The $\pi / 2$ dephasing between the $x$ and $y$ oscillations in this nonplanar motion is independent of the relative phase or amplitude of the $x$ and $y$ driving, as circularly polarized response can be obtained with linearly polarized driving. This circular polarization should be observed for high quality NNs and it represents a third mechanical regime beyond the linear and the Duffing linearly polarized responses for all regular polygon section rods because their linear polarizations are degenerate. We are currently analyzing the potential application to NEMS gyroscopes.

\section{ACKNOWLEDGMENTS}

The authors thank O. Thomas, E. de Langre, and A. Lazarus for fruitful discussions. This work is supported by the French National Research Agency (ANR) through the Nanoscience and Nanotechnology Program (Project NEXTNEMS No. ANR-07-NANO-008-01).

\footnotetext{
*sorin.perisanu@univ-lyon1.fr

†pascal.vincent@univ-lyon1.fr

${ }^{1}$ J. Yang, T. Ono, and M. Esashi, Appl. Phys. Lett. 77, 3860 (2000).

${ }^{2}$ S. Perisanu, P. Vincent, A. Ayari, M. Choueib, S. T. Purcell,
}

M. Bechelany, and D. Cornu, Appl. Phys. Lett. 90, 043113 (2007).

${ }^{3}$ Z. Chen, J. Appenzeller, Y. M. Lin, J. Sippel-Oakley, A. G. Rinzler, J. Tang, S. J. Wind, P. M. Solomon, and P. Avouris, Science 311, 1735 (2006). 
${ }^{4}$ K. Jensen, K. Kim, and A. Zettl, Nat. Nanotechnol. 3, 533 (2008).

${ }^{5}$ B. Lassagne, D. Garcia-Sanchez, A. Aguasca, and A. Bachtold, Nano Lett. 8, 3735 (2008).

${ }^{6}$ H.-Y. Chiu, P. Hung, H. W. Ch. Postma, and M. Bockrath, Nano Lett. 8, 4342 (2008).

${ }^{7}$ W. Zhang, R. Baskaran, and K. L. Turner, Sens. Actuators, A 102, 139 (2002).

${ }^{8}$ A. Ayari, P. Vincent, S. Perisanu, M. Choueib, V. Gouttenoire, M. Bechelany, D. Cornu, and S. T. Purcell, Nano Lett. 7, 2252 (2007).

${ }^{9}$ H. S. Kim, H. Qin, M. S. Westphall, L. M. Smith, and R. H. Blick, Nanotechnology 18, 065201 (2007).

${ }^{10}$ B. Lassagne, Y. Tarakanov, J. Kinaret, D. Garcia-Sanchez, and A. Bachtold, Science 325, 1107 (2009).

${ }^{11}$ G. A. Steele, A. K. Hüttel, B. Witkamp, M. Poot, H. B. Meerwaldt, L. P. Kouwenhoven, and H. S. J. van der Zant, Science 325, 1103 (2009).

${ }^{12}$ S. Sapmaz, Ya. M. Blanter, L. Gurevich, and H. S. J. van der Zant, Phys. Rev. B 67, 235414 (2003).

${ }^{13}$ A. H. Nayfeh and P. F. Pai, Int. J. Non-linear Mech. 24, 139 (1989).

${ }^{14}$ P. F. Pai and A. H. Nayfeh, Int. J. Non-linear Mech. 25, 455 (1990).
${ }^{15}$ W. G. Conley, A. Raman, C. M. Krousgrill, and S. Mohammadi, Nano Lett. 8, 1590 (2008).

${ }^{16}$ J. P. Cusumano and F. C. Moon, J. Sound Vib. 179, 185 (1995).

${ }^{17}$ S. Perisanu, P. Vincent, A. Ayari, M. Choueib, D. Guillot, M. Bechelany, D. Cornu, P. Miele, and S. T. Purcell, Phys. Status Solidi A 204, 1645 (2007).

${ }^{18}$ S. T. Purcell, P. Vincent, C. Journet, and V. T. Binh, Phys. Rev. Lett. 89, 276103 (2002).

${ }^{19}$ See supplementary material at http://link.aps.org/supplemental/ 10.1103/PhysRevB.81.165440 for setup description and experimental/simulated films.

${ }^{20}$ L. D. Landau and E. M. Lifshitz, Theory of Elasticity (Butterworth Heinemann, Oxford, England, 1986).

${ }^{21} m_{1}=\int_{0}^{1} f^{2}(s) d s, m_{2}=\int_{0}^{1}\left[\int_{0}^{s} f^{\prime 2}\left(s_{1}\right) d s_{1}\right]^{2} d s, k_{1}=\int_{0}^{1} f^{\prime \prime 2}(s) d s$, and $k_{2}$ $=\int_{0}^{1} f^{\prime 2}(s) f^{\prime \prime 2}(s) d s$.

${ }^{22}$ P. Poncharal, Z. L. Wang, D. Ugarte, and W. A. de Heer, Science 283, 1513 (1999).

${ }^{23}$ V. Sazonova, Y. Yaish, H. Üstünel, D. Roundy, T. A. Arias, and P. L. McEuen, Nature (London) 431, 284 (2004).

${ }^{24}$ K. L. Ekinci and M. L. Roukes, Rev. Sci. Instrum. 76, 061101 (2005).

${ }^{25}$ S. Perisanu, V. Gouttenoire, P. Vincent, A. Ayari, M. Choueib, M. Bechelany, D. Cornu, and S. T. Purcell, Phys. Rev. B 77, 165434 (2008). 